\title{
BIOPHARMACEUTICS AND PHARMACOKINETICS OF [D-ALA2, D- LEU5]ENKEPHALIN AFTER VARIOUS ROUTES OF ADMINISTRATION
}

\author{
H. J. Lee, G. L. Amidon, and L. Y. Yek \\ TSRL Inc. and College of Pharmacy, The University of Michigan \\ 540 Avis Dr., Ann Arbor, Ml. 48108
}

The biopharmaceutics and pharmacokinetics of [D-ala2, D-leu5]enkephalin (YdAGFdL) was investigated after six intravenous, oral, and nasal administration in a randomized cross-over fashion in two dogs. Following administration of [3H]YdAGFdL, blood samples were collected from the forearm vein over $90 \mathrm{~min}$ and assayed by radiometric thin layer chromatography for intact YdAGFdL and tyrosine (metabolite), respectively. Data were analyzed by non-compartmental pharmacokinetic methods. For each study, the areas under $Y d A G F d L$ and tyrosine curves (AUC) from time zero to 90 min were estimated by the trapezoidal rule. After intravenous dosings of $2.8 \mathrm{ug}$, total body clearance, volume of distribution at steady state, half-life of elimination phase, and mean residence time were $0.65 \pm 0.16$ (S.E.) $\mathrm{l} / \mathrm{min}, 6.95 \pm 1.21 \mathrm{l}, 22.3 \pm 3.4 \mathrm{~min}$, and $11.7 \pm 1.6 \mathrm{~min}$, respectively. Time to reach the maximum concentration (Tmax) for YdAGFdL after nasal administration was $7.3 \pm 0.8 \mathrm{~min}$. The Tmax for tyrosine after intravenous, oral, and nasal administrations were $5.4 \pm 2.5,9.7 \pm 2.3$, and $11.4 \pm 3.4$ min, respectively. Mean bioavailability after oral $(11.2 \mathrm{ug})$ and nasal $(2.8 \mathrm{ug})$ administration were less than $0.5 \%$ (based on the detection limit) and $5.9 \pm 4.8 \%$, respectively. The fractions of the tyrosine AUC over the AUC of total radioactivity in blood following intravenous, oral, and nasal administration were $0.071,0.137$, and 0.048 , respectively. These results indicate that even the 'stabilized' peptide is extensively metabolized during absorption in the Gl tract resulting in the poor oral systemic availability. The absorption of small peptide drugs could be improved significantly $(p<0.05)$ after nasal administration. Application of these results to the delivery of peptide drugs will be discussed. 